\title{
BURNOUT EN PROFESIONALES DE LA SALUD EN CONTEXTO DE PANDEMIA: UNA PROPUESTA METODOLÓGICA PARA LA DETECCIÓN DE PATRONES BASADA EN INTELIGENCIA ARTIFICIAL
}

\author{
Alejandro Zamudio Sosa, Samuel Ángel Rojo López, \\ Fernando Fuentes Gallegos y Jorge Iván Fletes Gutiérrez \\ UNAM, UAQ, UFRO, UdeG \\ México
}

\section{RESUMEN}

\begin{abstract}
Aunque los índices de desgaste profesional o burnout pueden variar en función de organizaciones, climas laborales o diversos tipos de trabajadores, varias investigaciones reportan índices de prevalencia superiores en la población médica y profesionales de la salud en comparación con otras poblaciones (Adán, Jiménez y Herrera, 2004; Hernández, 2003; Toral-Villanueva, Aguilar-Madrid y Juárez-Pérez, 2009; Breilh, 1993); aunado a esto, investigaciones han reportado un aumento de burnout en dichos profesionales en el contexto de la pandemia ocasionada por el Covid-19 (Morgantini et al., 2020). Por esta razón y con el objetivo de detectar patrones en cuanto a variables sociodemográficas, de carga laboral y de nivel de burnout, y contar con un clasificador para nuevos profesionales para la planeación de intervenciones, se implementaron algoritmos de inteligencia artificial en una muestra no probabilística a 724 médicos y enfermeros de México mediante formularios de Google y el uso de la herramienta de Facebook Ads. El formulario aplicado contenía el Maslach Burnout Inventory (MBI) (Maslach y Jackson, 1981), para evaluar el burnout y adaptado a población mexicana (Beltrán, Moreno y Estrada, 2016), así como preguntas adicionales relacionadas a variables sociodemográficas y carga laboral en el contexto de la pandemia ocasionada por el Covid-19. Se implementó el algoritmo de clusterización Kamila y se encontraron cinco grupos de profesionales con características particulares entre cada uno de ellos; finalmente se entrenó un modelo de clasificación utilizando un algoritmo de SVM (Support Vector Machine) con kernel gaussiano para clasificar nuevas observaciones en algún grupo resultado de la etapa clusterización, el cual tuvo una precisión de 0.92. La aplicación de algoritmos basados en inteligencia artificial puede servir para planear y desarrollar intervenciones grupales lo más especializadas posible y crear plataformas que arrojen recomendaciones psicológicas personalizadas a dichos profesionales.
\end{abstract}

Palabras Clave:

burnout, profesionales de la salud, inteligencia artificial, intervención.

\section{BURNOUT IN HEALTH PROFESSIONALS IN THE CONTEXT OF A PANDEMIC:}

\section{A METHODOLOGICAL PROPOSAL FOR THE DETECTION OF PATTERNS BASED ON ARTIFICIAL}

\section{ABSTRACT}

\author{
INTELLIGENCE
}

Although the rates of professional attrition or burnout may vary depending on organizational contexts and different types of workers, several investigations report higher prevalence rates in the medical and health professional population compared to other populations (Adán, Jiménez y Herrera, 2004; Hernández, 2003; Toral-Villanueva et al., 2009; Breilh, 1993), in addition to this, research has reported an increase in burnout in these professionals in the context of the pandemic caused by Covid-19 (Morgantini et al., 2020). For this reason and with the aim of detecting patterns in terms of sociodemographic variables, workload and burnout level and having a classifier for new professionals, artificial intelligence algorithms were implemented in a non-probabilistic sample to 724 doctors and nurses from Mexico through Google forms and the use of the Facebook Ads tool. The applied form contained the Maslach Burnout Inventory (MBI) (Maslach y Jackson, 1981), to assess burnout and adapted to the Mexican population (Beltrán, Moreno y Estrada, 2016), as well as additional questions related to sociodemographic variables and workload in the context of the pandemic caused by Covid-19. Subsequently, the Kamila clustering algorithm was implemented and 5 groups of professionals with particular characteristics were found among each of them, finally a classification model was trained using an SVM (Support Vector Machine) algorithm with Gaussian kernel to classify new observations in some group resulting from the clustering stage, which had a precision of 0.92. The application of algorithms based on artificial intelligence can be used to plan and carry out group interventions that are as specialized as possible and create platforms that can deliver personalized psychological recommendations to these professionals.

Keywords:

burnout, health professionals, artificial intelligence, intervention.

Bitácora del Artículo:

| Recibido: 8 de Febrero de 2021 | Aceptado: 04 abril de 2021 | Publicado en línea: Julio - Diciembre de 2021 | 
Artículo empírico | Burnout en profesionales de la salud...| Zamudio-Sosa, Rojo-López, Fuentes-Gallegos y Fletes-Gutiérrez

\title{
Autoría y Derechos de Propiedad Intelectual
}

\section{BURNOUT EN PROFESIONALES DE LA SALUD EN CONTEXTO DE PANDEMIA: UNA PROPUESTA METODOLÓGICA PARA LA DETECCIÓN DE PATRONES}

\author{
BASADA EN INTELIGENCIA ARTIFICIAL
}

Alejandro Zamudio Sosa, Samuel Ángel Rojo López, Fernando Fuentes Gallegos y Jorge Iván, Fletes Gutiérrez

UNAM

México

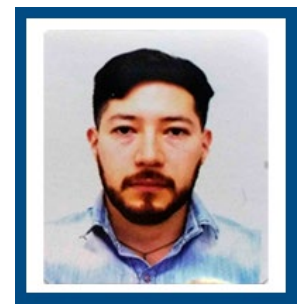

Alejandro Zamudio Sosa

Facultad de Psicología, UNAM

Correo: zamudiososaalejandro@gmail.com

Estudiante de doctorado en Psicología Social de la Universidad Nacional Autónoma de México, actualmente desarrolla proyectos de investigación utilizando herramientas de ciencia de datos e inteligencia artificial para el análisis de datos psicológicos y de ciencias sociales.

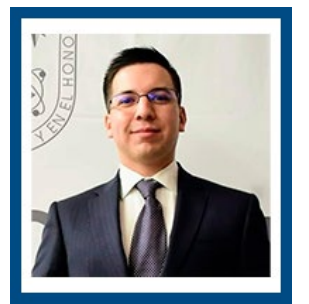

Samuel Ángel Rojo López

Independiente

Correo:rl.samuel.an@gmail.com

Ingeniero en Biomédica por la Universidad Autónoma de Querétaro con un diplomado en Tecnología de Biosensores por la misma institución, actualmente trabaja como Ingeniero de Datos en una empresa consultora de tecnología.

\section{CONTRIBUCIÓN DE LOS AUTORES}

Alejandro Zamudio Sosa estuvo a cargo de la propuesta y coordinación del desarrollo de la misma. | Samuel Ángel Rojo López participó en la metodología, ingesta de datos, limpieza, análisis exploratorio y experimentación de agrupamiento y clasificación. | Fernando Fuentes Gallegos colaboró en la metodología, revisión bibliográfica, limpieza, análisis exploratorio y experimentación de agrupamiento y clasificación. | Jorge Iván Gutiérrez participó en la metodología, revisión bibliográfica, limpieza, análisis exploratorio y experimentación de agrupamiento y clasificación.

\section{AGRADECIMIENTOS}

Se agredece todas las personas involucradas en la realización de esta investigación.

\section{Datos de FiLIACIÓN DE los Autores}

Alejandro Zamudio Sosa está adscrito a la Facultad de Psicología, UNAM | Samuel Ángel Rojo López, Fernando Fuentes Gallegos y Jorge Iván, Fletes Gutiérrez son investigadores independientes.

Copyright: (c) 2021 Zamudio-Sosa, A.;Rojo-López, S.; Fuentes-Gallegos, F.; \& Gutiérrez, J. I.

Este es un artículo de acceso abierto distribuido bajo los términos de la licencia Creative Commons Reconocimiento-NoComercial 4.0 Internacional, por lo que su contenido gráfico y escrito se puede compartir, copiar y redistribuir total o parcialmente sin necesidad de permiso expreso de sus autoras con la única condición de que no se puede usar con fines directamente comerciales y los términos legales de cualquier trabajo derivado deben ser los mismos que se expresan en la presente declaración. La única condición es que se cite la fuente con referencia a la Revista Digital Internacional de Psicología y Ciencia Social y a sus autoras. 


\section{TABLA DE CONTENIDO}

NATURALEZA DE LA INNOVACIÓN

CARACTERÍSTICAS DE LA INNOVACIÓN

RANGO DE APLICACIÓN DE LA INNOVACIÓN

FUNCIONAMIENTO DE LA INNOVACIÓN

CONCLUSIONES 
E I impacto del estrés laboral en la salud mental es una preocupación creciente (Adán, Jiménez y Herrera, 2004). Específicamente en el ambiente hospitalario, Bianchini-Matamoros (1997) considera que la salud mental requiere una especial atención, pues considera que existen diferentes factores que pueden afectar la salud mental de estos profesionales; entre los principales factores que este autor considera se encuentran: 1) extensos horarios de trabajo; 2) atención a diferentes pacientes que pueden enfrentar crisis y familiares que enfrentan pérdidas, y 3) una creciente demanda de los servicios de salud, como la actual pandemia ocasionada por el Covid-19.

Así, uno de los principales problemas estudiados respecto al trabajo de profesionales de la salud ha sido el estrés laboral crónico, también conocido como "síndrome de burnout". Éste se considera una patología laboral ocasionada por el estrés constante al que es sometido cualquier profesional en su ámbito laboral. Dicho estrés tiende a ser crónico, causando trastornos psicosomáticos y de conducta (Aranda-Beltrán, Pando-Moreno, Torres-López, Salazar-Estrada y Franco-Chávez, 2005). Otros autores (Bianchini-Matamoros, 1997) consideran que el síndrome de burnout o estrés laboral es un estado emocional que derivada de una sobrecarga de estrés y que influye en la motivación, actitudes y conducta de los profesionales que lo padecen. Este estrés crónico casi siempre es acompañado de síntomas somáticos, sentimientos de alienación, pérdida de motivación y sensación de fracaso.

Aunque los índices de desgaste profesional o burnout pueden variar en función del tipo de organización, ambiente laboral o profesiones específicas, varias investigaciones reportan niveles de prevalencia más altas en médicos y profesionales de la salud en comparación con la población de otros ámbitos laborales (Breilh, 1993; Adán, Jiménez y Herrera, 2004; Hernández, 2003; Toral-Villanueva et al., 2009).

Las consecuencias del burnout en los profesionales de la salud son amplias y afectan la salud mental, física, la calidad de vida y la eficacia de estos trabajadores. Al mismo tiempo puede generar una deficiente atención hacia el paciente y un deterioro en el sistema de salud (Bianchini, 1997). Aunado a esto, Adán, Jiménez y Herrera (2004) consideran que dichos profesionales no buscan evaluar ni atender su estrés de manera profesional por temor a la estigmatización, a perder credibilidad frente a sus compañeros y pacientes, o al intentar ocultar problemas por miedo a perder el trabajo.

Asimismo hay evidencia para pensar que el burnout no tiene la misma naturaleza en todos los profesionales de la salud, la profesión específica (Schaufeli y Enzmann, 2020), el nivel de atención (Hernández, 2003), el sexo (Bernard y Meade, 1993; Elmore et al., 2016; Gil-Monte, 2002) y las horas trabajadas (Ávila-Toscano, Gómez-Hernández y Montiel-Salgado, 2010), entre otras variables (Toral-Villanueva et al., 2009), pueden incidir en la naturaleza y el nivel de burnout que pueden experimentar estos profesionales. Por tanto, es conveniente considerar dichas diferencias en la planeación y desarrollo de posibles intervenciones. Adicionalmente, varios estudios (Barello, Palamenghi y Graffigna, 2020; Morgantini et al., 2020) han reportado que la presión psicológica, síntomas somáticos y el burnout han aumentado en profesionales de la salud derivado a la sobrecarga laboral que implica la pandemia causada por el Covid-19. Otros autores (Sultana, Sharma, Hossain, Bhattacharya y Purohit, 2020) sugieren que el fenómeno de sobrecarga laboral ocasionado por la pandemia hace cada vez más urgente la necesidad de herramientas tecnológicas que ayuden a dichos profesionales a promover el autocuidado, el bienestar y el monitoreo de su salud mental.

Esta situación, y en el contexto de la pandemia causada por el Covid-19, plantea la necesidad de implementar acciones para buscar incidir en esta problemática. Una de las formas de enfrentar el problema es tener procedimientos que permitan conocer conjuntos de profesionales con características sociodemográficas y psicológicas similares para planear e implementar intervenciones con el menor costo y lo más personalizadas posible, y que sea de ayuda para obtener indicadores de burnout actualizados de las poblaciones de estudiantes y profesionistas de médicos y enfermeros que puedan servir a los directivos de clínicas y/o hospitales para tomar decisiones y mejorar las condiciones de dichos profesionales. Así, el propósito de este artículo es dar a conocer una metodología basada en inteligencia artificial que permita a los profesionales de la salud mental detectar patrones entre los profesionales de la salud (en cuanto a burnout y otras variables relevantes se refiere) y contar con clasificadores automáticos para nuevos profesionales y así poder planear intervenciones más eficaces con el menor costo posible. Se espera que los algoritmos de inteligencia artificial ayuden a encontrar patrones en una muestra de profesionales de la salud y al mismo tiempo poder clasificar nuevos profesionales no observados antes con métricas buenas o aceptables. 


\section{Naturaleza de LA INNOVACIÓN}

Dada la incidencia creciente del burnout en profesionales de la salud, aunado a la gran demanda y carga laboral que el fenómeno de la pandemia ha traído a los médicos y enfermeras de México, es necesario disponer de herramientas metodológicas que permitan planear e implementar intervenciones psicológicas lo más personalizadas posible para atender este síndrome en dicha población. Para ello se utilizaron algoritmos de inteligencia artificial para identificar conjuntos de profesionales de la salud que compartieran la mayor cantidad de características posibles, teniendo en cuenta sus niveles de burnout, sus características sociodemográficas y su carga laboral en el contexto de la pandemia, para luego clasificar de modo automático a los profesionales de salud en alguno de los grupos formados.

\section{Características de la innovación}

Mediante un muestro no probabilístico por conveniencia se aplicaron 724 formularios de Google, distribuidos por medio de Facebook Ads (se seccionó al público al cual sería dirigida la encuesta mediante palabras clave como "medicina", "enfermería", "pacientes", "facultad médica" y "facultad de enfermería"), en las cuales se recabaron datos de las variables sexo, edad, profesión, nivel de atención, grado académico, número de pacientes promedio en una jornada, número promedio de horas trabajadas en una semana durante la pandemia causada por el Covid-19, en conjunto con 22 reactivos del Maslach Burnout Inventory (MBI) (Maslach y Jackson, 1981), para evaluar el burnout en profesionales de la salud y adaptado a la población mexicana (Beltrán, Moreno y Estrada, 2016). En dicho formulario se presentó un consentimiento informado aclarando la confidencialidad de los datos, así como el propósito de obtención de los mismos; en caso de no otorgar el consentimiento, el formulario se terminó para dichos participantes. La aplicación fue durante las dos primeras semanas de agosto de 2020.

El MBI es un cuestionario para evaluar el burnout; es muy utilizado en el mundo por sus propiedades psicométricas (Gil-Monte y Peiró-Silla, 1997); dicho cuestionario evalúa el burnout en tres factores: 1) agotamiento emocional; 2) despersonalización, y 3) desrealización profesional. Para la limpieza de datos, extracción de características y creación de gráficas se usó el lenguaje Python, y para la implementación de los algoritmos se utilizó el lenguaje R. Se extrajeron indicadores de los tres componentes del burnout (agotamiento emocional, despersonalización y des- realización profesional) que mide el $\mathrm{MBI}$, mediante la sumatoria lineal de los reactivos 1 , 2, 3, 6, 8, 13, 14, 16 y 20 para el agotamiento emocional; los reactivos 5, 10, 11, 15 y 22 para el factor de despersonalización, y de los reactivos $4,7,9,12,17,18,19$ y 21 para la desrealización profesional. Para evaluar la calidad de la medición de burnout se hizo un análisis factorial confirmatorio mostrando ajustes adecuados (figura 1). Dado que en los tres componentes de burnout se relacionaron de manera lineal se decidió unir los tres factores en un solo factor global o acumulado de burnout para el resto del análisis.

Figura 1.

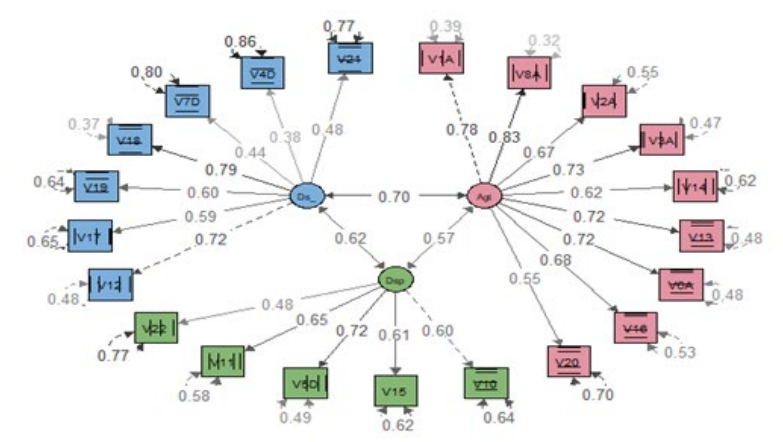

Análisis factorial confirmatorio de MBI, adaptado en población mexicana, que muestra adecuada estructura interna.

En cuanto a la descripción de la muestra, $81 \%$ de los encuestados corresponde a mujeres, $64 \%$ tenía la profesión de enfermería (figura 2); los principales estados donde vivían los participantes fueron Nuevo León (23\%), Ciudad de México (21\%), Estado de México (17\%) y Jalisco (17\%).

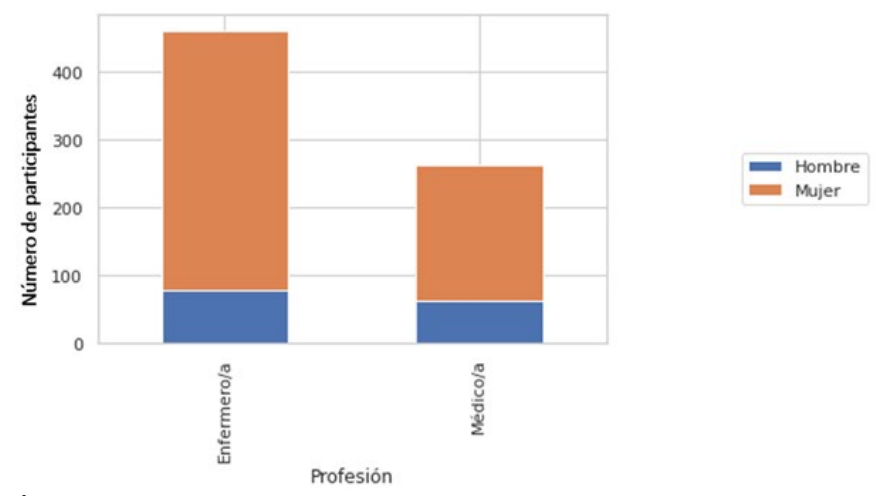

Figura 2.

Número de participantes por profesión y sexo.

En cuanto a la proporción por niveles de atención, ésta se mantuvo relativamente equilibrada entre los tres tipos de niveles de atención (figura 3); sin embargo, la proporción de estudiantes de especialidad y/o posgrado fue menor que cualquier otro grado.

Por otra parte, la edad promedio fue de 28.58 años con una desviación estándar de 6.71 años (figura 4). 


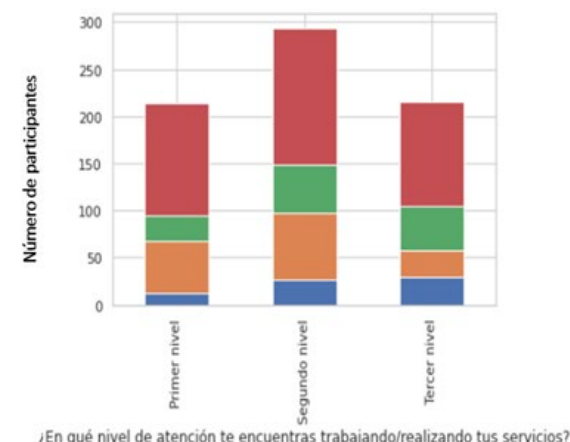

Figura 3.

Número de participantes por nivel de atención y grado académico.

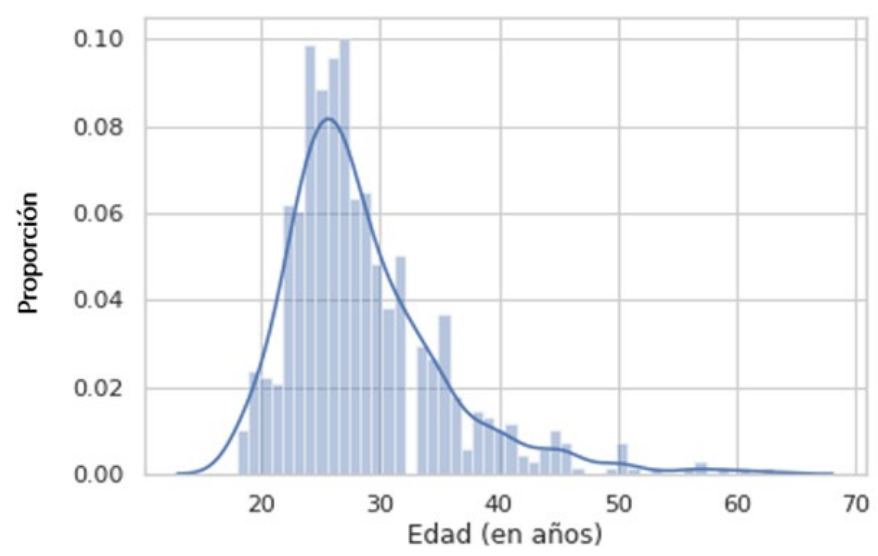

Figura 4.

Distribución de edad de los participantes.

Para el número de pacientes se encontró una media de 14.24 pacientes por jornada laboral con una desviación estándar de 12.62 pacientes (figura 5), para las horas de trabajo se obtuvo una media de 44.49 horas y una desviación estándar de 20.25 horas (figura 6). Por último, para el burnout total se obtuvo una media de 57.38 y una desviación estándar de 10.93 (con un rango de 25 a 110) (figura 7). Por último, en la figura 8 se muestra cómo el acumulado de burnout o burnout total se incrementa conforme aumentan las horas laborales y el número de pacientes, mientras que la edad parece desempeñar un papel secundario.

En segundo paso se procedió a aplicar el algoritmo de agrupamiento Kamila (Foss, Markatou, Ray y Heching, 2016) para la conformación de clústeres porque se contaba con una base de datos mixta, es decir, variables categóricas y variables continuas. Las variables contempladas para Kamila fueron edad, número de pacientes por jornada durante la pandemia, número de horas laborales a la semana, nivel de burnout global, sexo, nivel educativo, nivel de atención y profesión; dichas variables fueron seleccionas con base en las carac-

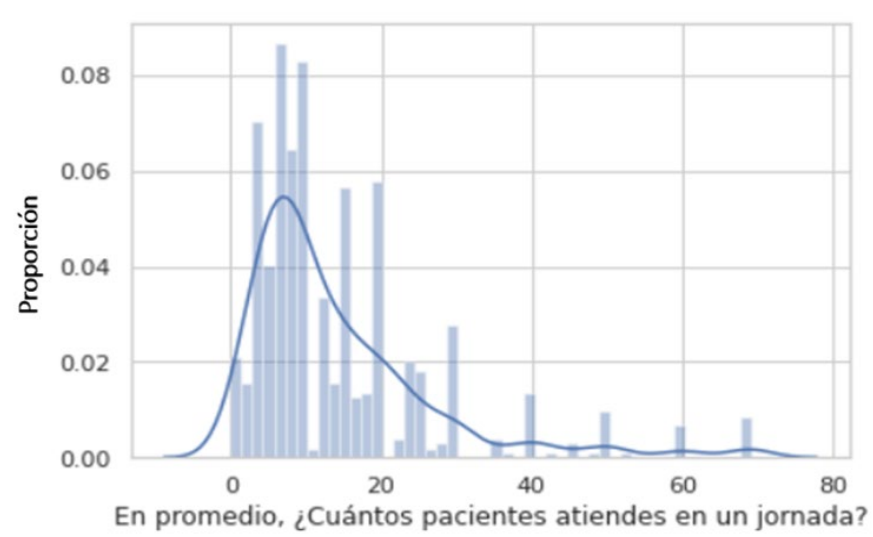

Figura 5.

Distribución de pacientes atendidos en una jornada laboral reportados por los participantes.

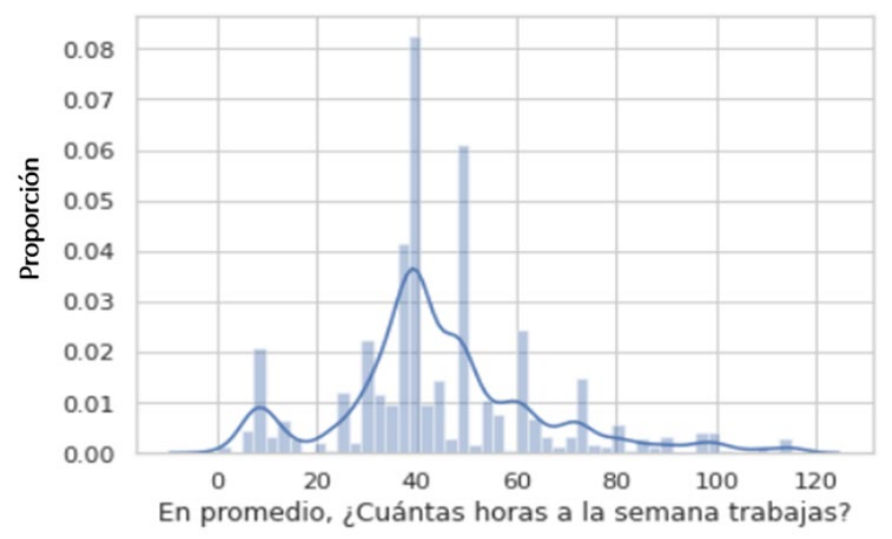

Figura 6.

Distribución de horas promedio trabajadas a la semana reportadas por los participantes.

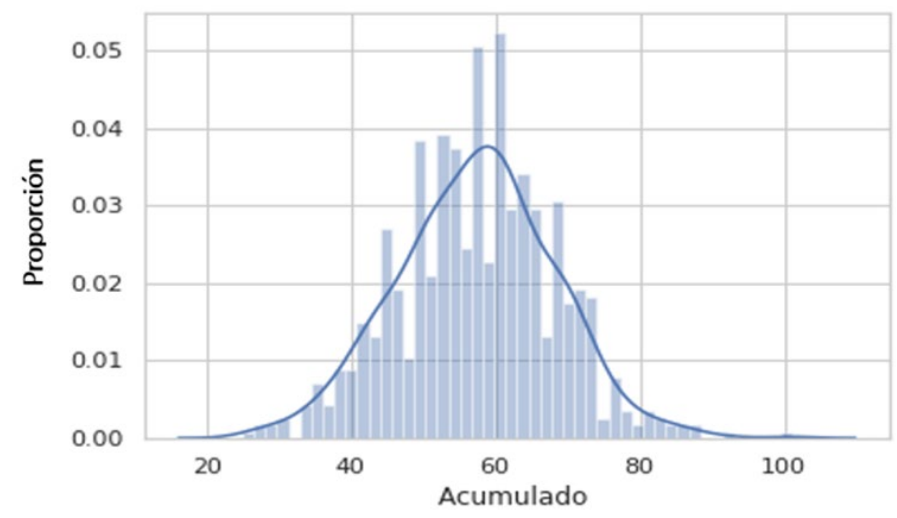

Figura 7.

Distribución de burnout total (acumulado) de los participantes, con un mínimo de 22 y un máximo de 110. 


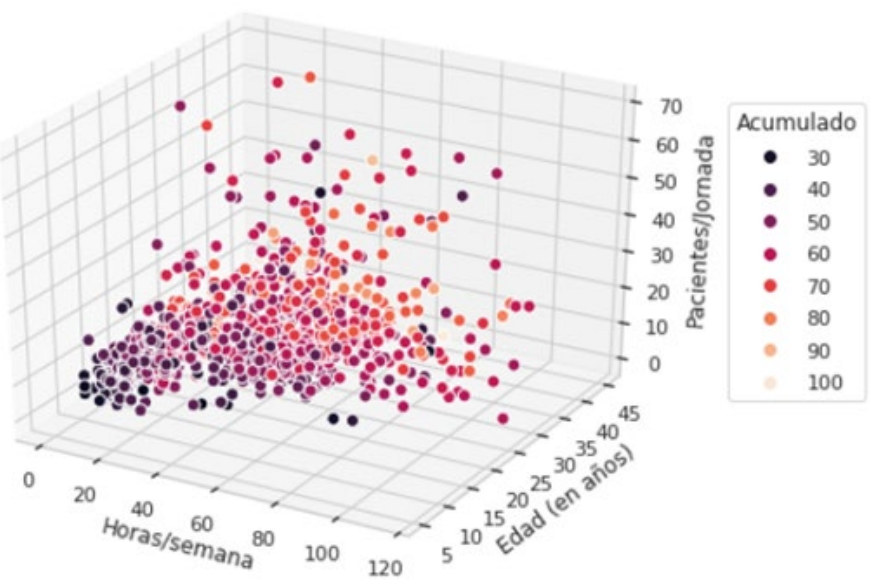

Figura 8.

Relación entre la edad, las horas trabajadas a la semana, el número de pacientes atendidos por jornada laboral y el acumulado o burnout total en los participantes. A medida que aumentan las horas trabajadas a la semana y el número de pacientes, mayor es el nivel de burnout acumulado.

terísticas relevantes reportadas en la literatura científica (Aranda-Beltrán et al., 2005; Contreras, Juárez y

Murrain, 2008). Aunque se obtuvo la métrica de fuerza predicción desde 2 hasta 10 clústeres como lo sugieren los autores (Foss et al., 2016), el número de clústeres se seleccionó sobre todo con base en la interpretabilidad de los grupos, es decir, se seleccionaron cinco clústeres, pues este número agrupó la máxima diferencia entre burnout y las demás variables contempladas en cada uno de los grupos (tablas 1-5).

\section{Tabla 1.}

Distribución de burnout total (acumulado) de los participantes, con un mínimo de 22 y un máximo de 110.

\begin{tabular}{|ccccc}
\hline CLÚSTER & EdAD & $\begin{array}{c}\text { NÚmERO DE } \\
\text { PACIENTES } \\
\text { ATENDIDOS } \\
\text { EN UNA } \\
\text { JORNADA } \\
\text { LABORAL }\end{array}$ & $\begin{array}{c}\text { NúMERO } \\
\text { DE HORAS } \\
\text { LABORALES }\end{array}$ & BURNOUT \\
\hline 1 & 26.89 & 7.92 & 46.56 & 61.67 \\
\hline 2 & 31.29 & 27.69 & 40.71 & 62.38 \\
\hline 3 & 31.38 & 10.57 & 38.85 & 46.01 \\
\hline 4 & 26.57 & 21.10 & 82.41 & 57.71 \\
\hline 5 & 26.53 & 11.59 & 12.49 & 56.58 \\
\hline
\end{tabular}

Tabla 2.

Proporción de sexo por cada clúster de profesionales de la salud.

\begin{tabular}{ccc} 
Clúster & Hombre & MujeR \\
\hline 1 & 0.19 & 0.81 \\
\hline 2 & 0.11 & 0.89 \\
\hline 3 & 0.25 & 0.75 \\
\hline 4 & 0.24 & 0.76 \\
\hline 5 & 0.27 & 0.73 \\
\hline
\end{tabular}

Tabla 3.

Proporción de profesión por cada clúster de profesionales de la salud.

\begin{tabular}{ccc} 
Clúster & Médico (A) & Enfermero (A) \\
\hline 1 & 0.90 & 0.10 \\
2 & 0.31 & 0.69 \\
3 & 0.56 & 0.44 \\
4 & 0.43 & 0.57 \\
5 & 0.66 & 0.34 \\
\hline
\end{tabular}

Tabla 4.

Proporción de nivel de atención por cada clúster de profesionales de la salud.

\begin{tabular}{|cccc|}
\hline Clúster & \multicolumn{3}{c}{ Nivel de atención } \\
\hline 1 & Primario & Secundario & Terciario \\
\hline 2 & 0.16 & 0.41 & 0.43 \\
\hline 3 & 0.62 & 0.30 & 0.08 \\
\hline 4 & 0.22 & 0.47 & 0.31 \\
\hline 5 & 0.27 & 0.46 & 0.28 \\
\hline
\end{tabular}

Tabla 5.

Proporción grado académico por cada clúster de profesional de la salud.

\begin{tabular}{|c|c|c|c|c|}
\hline \multirow[b]{2}{*}{ Clúster } & \multicolumn{4}{|c|}{ GRADO ACADÉMICO } \\
\hline & $\begin{array}{l}\text { ESTUDIANTE } \\
\text { LICENCIATURA }\end{array}$ & $\begin{array}{c}\text { TITULADO } \\
\text { LICENCIADO }\end{array}$ & $\begin{array}{c}\text { ESTUDIANTE } \\
\text { DE } \\
\text { POSGRADO }\end{array}$ & $\begin{array}{c}\text { TITULADO } \\
\text { DE } \\
\text { POSGRADO }\end{array}$ \\
\hline 1 & 0.09 & 0.15 & 0.11 & 0.65 \\
\hline 2 & 0.09 & 0.15 & 0.39 & 0.45 \\
\hline 3 & 0.08 & 0.20 & 0.29 & 0.43 \\
\hline 4 & 0.21 & 0.29 & 0.11 & 0.39 \\
\hline 5 & 0.08 & 0.44 & 0.04 & 0.44 \\
\hline
\end{tabular}

En la figura 9 se muestran los cinco clústeres formados por el algoritmo Kamila, graficándolos con base en el nivel de burnout, las horas trabajadas en una jornada y el 
número de pacientes atendidos durante la pandemia en una jornada. De manera resumida, los cinco grupos que se formaron en los clústeres tenían la siguiente naturaleza.

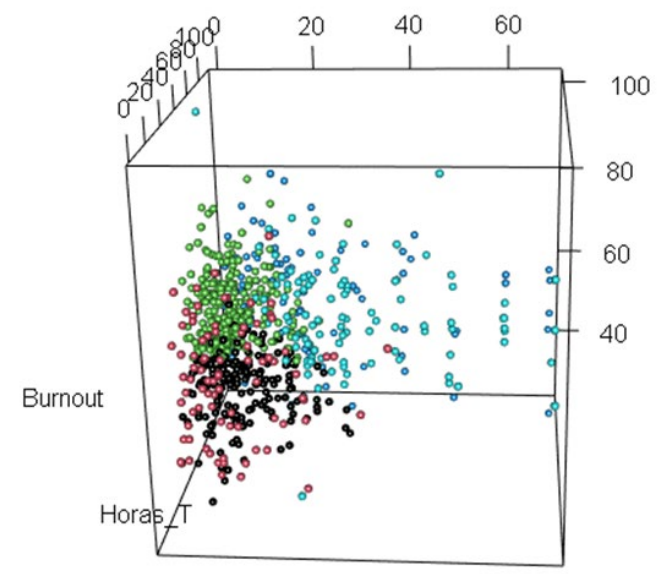

\section{Figura 9.}

N_pacientes

Representación en gráfico de 3D (con las variables de burnout total, número de pacientes y horas trabajadas en una jornada laboral) de los clústeres formados por el algoritmo Kamila.

1. El primer grupo se caracteriza por ser jóvenes (media de 26.8 años), atender pocos pacientes por jornada (media de 7.9 pacientes), tener media alta cantidad de horas de trabajo a la semana (media de 46.5 horas) y medio alto burnout (media de 61.6).

2. El segundo grupo lo conformaban adultos jóvenes (media de 31.2 años), con alta cantidad de pacientes atendidos por jornada (media de 27.6 pacientes), horas de trabajo promedio (media de 40.7 horas) y alto burnout (62.3).

3. El tercer grupo se conformó con adultos jóvenes (media de 31.3 años), con media baja cantidad de pacientes que atienden (10.5 pacientes), horas de trabajo medio bajas (media de 38.8 horas) y burnout bajo (media de 46).

4. El cuarto grupo se conformó con jóvenes (media de 26.5 años), media alta cantidad de pacientes atendidos (media de 21 pacientes), altas horas de trabajo (82.4 horas) y burnout medio (media de 57.7).

5. El último grupo se formó de jóvenes (media de 26.5 años), medio bajo pacientes (media de 11.5 pacientes), bajas horas de trabajo (media de 12.4 horas), medio bajo burnout (media de 56.5).

En un tercer paso se aplicó el algoritmo de SVM con kernel gaussiano para clasificación, se dividió la base de datos en $70 \%$ para entrenar y $30 \%$ para someter a prueba el algoritmo de clasificación, y se obtuvo una matriz de confusión (tabla 6). Después se hizo una validación cruzada con cuatro folds para evaluar la precisión del modelo de clasificación utilizado, obteniendo un promedio de 0.92 (0.87-0.95 grados de libertad a $95 \%$ ) con una desviación estándar de 0.0230. Para finalizar, se obtuvieron métricas de sensibilidad, especificidad, accuracy por clase (tabla 7).

Tabla 6.

Matriz de confusión utilizada para comparar los casos asignados a cada clúster por el algoritmo de clasificación por los casos reales en cada clúster.

\begin{tabular}{cccccc|}
$\begin{array}{c}\text { PredicCión } \\
\text { HECHA POR EL } \\
\text { CLASIFICADOR }\end{array}$ & $\mathbf{1}$ & $\mathbf{2}$ & $\mathbf{3}$ & $\mathbf{4}$ & $\mathbf{5}$ \\
\hline 1 & 56 & 2 & 0 & 1 & 1 \\
\hline 2 & 3 & 67 & 3 & 0 & 2 \\
\hline 3 & 0 & 0 & 20 & 0 & 0 \\
\hline 4 & 0 & 0 & 0 & 22 & 0 \\
\hline 5 & 1 & 1 & 2 & 1 & 35 \\
\hline
\end{tabular}

Tabla 7.

Estadísticas de sensibilidad, especificidad y precisión por cada clúster obtenidas por el clasificador empleado.

\begin{tabular}{lccccc} 
& \multicolumn{5}{c}{ CLÚSTER } \\
& $\mathbf{1}$ & $\mathbf{2}$ & $\mathbf{3}$ & $\mathbf{4}$ & $\mathbf{5}$ \\
\hline Sensibilidad & 0.93 & 0.96 & 0.80 & 0.92 & 0.92 \\
\hline Especificidad & 0.97 & 0.95 & 1.00 & 1.00 & 0.97 \\
\hline Precisión & 0.95 & 0.95 & 0.90 & 0.96 & 0.95
\end{tabular}

\section{RANGO de APLICACIÓN DE LA INNOVACIÓN}

Se considera que las jornadas laborales en México son de las más prolongadas en el mundo (Infobae, 2020), al mismo tiempo que las vacaciones en México son de las más breves (Animal Político, 2017); además, de acuerdo con algunas investigaciones recientes (La Jornada, 2020; Business Insider, 2020), la pandemia y el fenómeno de home office ha extendido las horas laborales de los trabajadores mexicanos, y en particular en profesionales de la salud (Barello et al., 2020; Morgantini et al., 2020). De este modo se puede suponer que el desgaste laboral no sólo afecta a los profesionales de la salud, pues múltiples sectores industriales y de servicios pueden estar gravemente afectados por dicho desgaste.

Respecto a lo anterior, en octubre de 2018 entró en vigor la Norma Oficial Mexicana NOM-035 (Diario Oficial de la Federación, 2018) que busca proteger del estrés laboral y de riesgos psicosociales al trabajador. Di- 
cha norma establece como obligación de las empresas la implementación de políticas de prevención de riesgos psicosociales, la identificación de trabajadores que hayan experimentado acontecimientos traumáticos por o durante el trabajo para canalizarlos y desarrollar actividades de prevención de dichos riesgos. El panorama planteado evidencia la necesidad de tener herramientas para identificar y atender condiciones relacionadas con el desgaste laboral en México. De esta manera, la metodología planteada puede ayudar a los profesionales de la salud mental a diseñar e implementar intervenciones con bajo costo, pues permite identificar conjuntos de profesionales y trabajadores con características similares (sociales y psicológicas) para facilitar el diseño de intervenciones grupales, talleres o cursos enfocados a las necesidades específicas de dichos grupos. Es decir, dicha metodología puede ayudar a crear aplicaciones para ofrecer recomendaciones personalizadas a los profesionales de la salud de prácticas de autociudado o meditación (Sultana et al., 2020), así como contar con una herramienta que permita clasificar a nuevos trabajadores para integrarlos a los grupos adecuados para su posible intervención. Aunado a esto, la implementación de este tipo de algoritmos de inteligencia artificial puede ayudar a generar reportes acerca de la incidencia y naturaleza de los diferentes trastornos en hospitales e instituciones y ayudar a los directivos a tomar decisiones para atender dichos trastornos.

\section{Funcionamiento de LA INNOVACIÓN}

En el contexto hospitalario, en muchas ocasiones los psicólogos clínicos tienen la responsabilidad de detectar y atender trastornos como depresión, ansiedad o burnout entre los profesionales de la salud; a su vez, en múltiples empresas cada vez es más frecuente que psicólogos tengan la responsabilidad de detectar y atender diferentes trastornos con tiempo y recursos limitados. Gil-Monte (2001) considera que la primera medida para atender el burnout en estos profesionales consiste en que éstos sean conscientes de las manifestaciones del síndrome; aunado a esto, son de gran ayuda intervenciones basadas en procesos cognitivos de autoevaluación y de tipo conductual para mitigar el estrés; al mismo tiempo es de utilidad promover estrategias de afrontamiento enfocadas en el problema y no de evitación, potenciar las habilidades sociales y eliminar o disminuir en lo posible los estresores a nivel organizacional. Gil-Monte también considera que es importante aumentar el asertividad, establecer objetivos factibles de conseguir, fomentar el apoyo emocional entre compañeros de trabajo y mejorar el clima organizacional.
De este modo la metodología planteada puede ayudar a los profesionales de la salud mental a detectar patrones clave para la correcta planeación e implementación de talleres o pláticas que fomenten dichas estrategias formando grupos con características similares y poder realizar intervenciones grupales con base en sus características; al mismo tiempo puede ayudar a elaborar informes para que directivos puedan definir acciones y modificar el clima organizacional con base en datos de las características de dichos grupos.

Con base en la naturaleza de los clústeres encontrados, se propone la creación de talleres enfocados en la mitigación del burnout, como técnicas de respiración, meditación y tiempo fuera (Sultana et al., 2020) para el primer y segundo grupos (que fueron los que presentaron índices más elevados de burnout). Para el tercer grupo, quienes presentaban menor burnout y al mismo tiempo mayor edad, se recomienda enfocar los talleres y pláticas en el acompañamiento emocional de los profesionales de la salud con quienes laboran; este grupo pudiera fungir de apoyo social para sus compañeros de trabajo con más burnout. Para el cuarto grupo se recomienda buscar apoyo de los puestos directivos y los tomadores de decisiones para disminuir la carga de trabajo y las horas laborales. De este modo, con base en las características de los grupos identificados se pueden implementar acciones conjuntas o separadas para atender las necesidades de cada uno de ellos.

\section{Conclusiones}

El burnout o desgaste laboral es un fenómeno que puede afectar de manera grave en diversos ámbitos de la vida de los profesionales de la salud que lo padecen, teniendo como consecuencias una disminución del desempeño laboral, mala satisfacción con su práctica, actitudes negativas hacia el cuidado de los pacientes (Hernández, 2003), conflictos laborales frecuentes, e incluso causar síntomas de depresión y/o ansiedad. Para ayudar a atender esta problemática es urgente contar con herramientas que puedan ayudar a detectar la naturaleza del burnout y su relación con otro tipo de variables, y que a la vez sean de fácil acceso y bajo costo para las instituciones.

En este sentido, el proyecto presentado logró recolectar información clave que permitió: 1) implementar un algoritmo que agrupara a los profesionales de la salud con base en sus características (psicológicas, de trabajo y sociodemográficas), y que dichos grupos fueran interpretables psicológicamente hablando, y 2) implementar un algoritmo que aprendiera a clasificar de manera correcta a nuevos profesionales. Lo anterior con

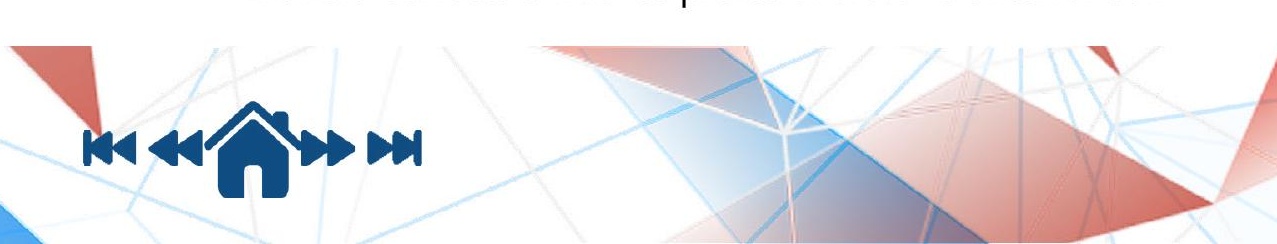


el objetivo planear e implementar intervenciones psicológicas, como talleres o pláticas, lo más personalizadas posible, a cada uno del grupo. Asimismo se recomienda aplicar la metodología presentada por hospital o laboratorio y así contar con patrones específicos por hospital y que sea de mayor ayuda para los profesionales de la salud mental encargados de los mismos.

Como pasos a seguir se propone que la herramienta considere variables que no fueron identificadas en este proyecto, como el clima laboral y el tipo de área clínica en que se trabaja; estos factores pueden ser relevantes, tal como se propone en estudios en otros países (Attia-Bari, Haroon y Bano, 2019). También se propone elaborar un dashboard (o tablero cuyo propósito es representar de manera visual indicadores para la toma de decisiones) con los resultados de la presente metodología para contar en tiempo real con indicadores de burnout y otro tipo de datos que permitan tomar decisiones basadas en datos. Asimismo es importante tener en cuenta profesionales administrativos de las clínicas u hospitales porque estos trabajadores también son susceptibles a presentar afectaciones por desgaste en el trabajo (Flores-Tesillo y Ruíz-Martínez, 2018).

El uso de inteligencia artificial puede ayudar a los profesionales de la salud mental a encontrar patrones en grandes cantidades de datos, patrones que pueden ayudar a conocer la naturaleza de los trastornos y al mismo tiempo tener más información para la implementación de intervenciones; esto resulta útil en épocas de pandemias, de acuerdo con la relativa rapidez con que pueden surgir problemas psicológicos asociados a la carga de trabajo en profesionales de la salud. El siguiente paso de este proyecto se enfocará en crear una plataforma y aplicación que pueda clasificar de modo automático a profesionales de la salud y proporcionar recomendaciones psicológicas lo más personalizadas posible, así como crear indicadores y visualizaciones de los datos recabados en poco tiempo.

Es importante destacar que los resultados obtenidos en esta metodología no son representativos de alguna población específica; es necesario que se aplique el presente procedimiento con muestras representativas o la población a la que se planearán las intervenciones (hospitales o clínicas completas); sin embargo, la facilidad y bajo costo del mismo (dado que se ocuparon exclusivamente herramientas y softwares gratuitos) permite su implementación de manera generalizada.

\section{Referencias}

Adán, J. C. M., Jiménez, B. M., \& Herrera, M. G. (2004). Desgaste profesional y salud de los profesionales médicos:
Revisión y propuestas de prevención. Medicina clínica, 123(7), 265-270. https://doi.org/10.1016/S00257753(04)74484-X

Animal Político (2017, julio 21). Mucho trabajo pocas vacaciones: México es uno de los países que menos días da a sus empleados. Recuperado el 30 de noviembre de 2020, de: https://www.animalpolitico.com/2017/07/vacacionesmexico-empleados/

Aranda-Beltrán, C., Pando-Moreno, M., Torres-López, T., Salazar-Estrada, J., \& Franco-Chávez, S. (2005). Factores psicosociales y síndrome de burnout en médicos de familia. Anales de la Facultad de Medicina, 66(3), 225-231.

Attia-Bari, R. K., Haroon, F., \& Bano, I. (2019). Burnout among pediatric residents and junior consultants working at a tertiary care hospital. Pakistan journal of medical sciences, 35(1), 45. https://doi.org/10.12669/pjms.35.1.43

Ávila-Toscano, J. H., Gómez-Hernández, L. T., \& Montiel-Salgado, M. M. (2010). Características demográficas y laborales asociadas al Síndrome de Burnout en profesionales de la salud. Pensamiento Psicológico, 8(15), 39-52.

Barello, S., Palamenghi, L., \& Graffigna, G. (2020). Burnout and somatic symptoms among frontline healthcare professionals at the peak of the Italian Covid-19 pandemic. Psychiatry research, 290, 113129. https://doi. org/10.1016/j.psychres.2020.113129

Beltrán, C. A., Moreno, M. P., \& Estrada, J. G. S. (2016). Confiabilidad y validación de la escala Maslach Burnout Inventory (Hss) en trabajadores del occidente de México. Salud Uninorte, 32(2), 218-227. https://doi. org/10.14482/sun.32.2.8828

Bernard, M., \& Meade, K. (eds.) (1993). Women come of age: perspectives on the lives of older women. London: $\mathrm{E}$. Arnold.

Bianchini-Matamoros, M. (1997). El Síndrome del Burnout en personal profesional de la salud. Medicina Legal de Costa Rica, 13(2-1-2), 189-192.

Breilh, J. (1993). Trabajo hospitalario, estrés y sufrimiento mental. Salud problema, 23, 21-37.

Business Insider (2020, 5 de agosto). Muchas reuniones y $48 \mathrm{~min}$ más: Así ha cambiado la jornada laboral desde que inicio la pandemia. Recuperado el 30 de noviembre de 2020, de: https://www-businessinsider-es.cdn.ampproject. org/c/s/www.businessinsider.es/jornada-laboral-dura48-minutos-pandemia-689893?amp

Contreras, F., Juárez, F., \& Murrain, E. (2008). Influencia del Burnout, la calidad de vida y los factores socioeconómicos en las estrategias de afrontamiento utilizadas por los profesionales y auxiliares de enfermería. Pensamiento psicológico, 4(11), 29-43.

Diario Oficial de la Federación (2018, 23 de octubre). Norma Oficial Mexicana NOM-035-STPS-2018, Factores de riesgo psicosocial en el trabajo-Identificación, análisis y prevención. Recuperado el 30 de noviembre de 2020: DOF - Diario Oficial de la Federación

Elmore, L. C., Jeffe, D. B., Jin, L., Awad, M. M., \& Turnbull, I. R. (2016). National survey of burnout among US general surgery residents. Journal of the American College of Surgeons, 223(3), 440-451.

Flores-Tesillo, S. Y., \& Ruíz-Martínez, A. O. (2018). Burnout en 
profesionales de la salud y personal administrativo en una unidad médica de primer nivel. Psicología y Salud, 28(1), 63-72. https://doi.org/10.25009/pys.v28i1.2539

Foss, A., Markatou, M., Ray, B., \& Heching, A. (2016). A semiparametric method for clustering mixed data. Machine Learning, 105(3), 419-458. https://doi. org/10.1007/s10994-016-5575-7

Gil-Monte, P. R. (2002). Influencia del género sobre el proceso de desarrollo del síndrome de quemarse por el trabajo (burnout) en profesionales de enfermería. Psicologia em estudo, 7(1), 3-10. https://doi.org/10.1590/S141373722002000100003

Gil-Monte, P. R. (2001). El síndrome de quemarse por el trabajo (síndrome de burnout): Aproximaciones teóricas para su explicación y recomendaciones para la intervención. Revista Psicologia Cientifica, 3(5).

Gil-Monte. P. R., \& Peiró-Silla, J. M. (1997). Desgaste psíquico en el trabajo: El síndrome de quemarse. Madrid: Síntesis.

Hernández, J. R. (2003). Estrés y burnout en profesionales de la salud de los niveles primario y secundario de atención. Revista cubana de salud pública, 29(2), 103-110.

Infobae (2020, 11 de febrero). La jornada laboral de los mexicanos es una de las más extensas del mundo. Recuperado el 30 de noviembre de 2020, de: https:// www.infobae.com/america/mexico/2020/02/11/lajornada-laboral-de-los-mexicanos-es-una-de-las-masextendidas-del-mundo/
La Jornada (2020, 6 de julio). Advierten abusos de laborar en casa. Recuperado el 30 de noviembre de 2020, de https:// www.jornada.com.mx/ultimas/sociedad/2020/07/06/ advierten-de-abusos-al-laborar-en-casa-9804.html

Maslach, C., \& Jackson, S. E. (1981). MBI: Maslach Burnout Inventory Manual. Palo Alto: University of California, Consulting Psychologists Press.

Morgantini, L. A., Naha, U., Wang, H., Francavilla, S., Acar, Ö., Flores, J. M., ... \& Weine, S. M. (2020). Factors contributing to healthcare professional burnout during the Covid-19 pandemic: A rapid turnaround global survey. PloS one, 15(9), e0238217. https://doi.org/10.1371/journal. pone. 0238217

Schaufeli, W., \& Enzmann, D. (2020). The Burnout Companion to Study and Practice: A Critical Analysis (Issues in Occupational Health) (English Edition) (1a. ed.). CRC Press. https://doi.org/10.1201/9781003062745

Sultana, A., Sharma, R., Hossain, M. M., Bhattacharya, S., \& Purohit, N. (2020). Burnout among healthcare providers during Covid-19 pandemic: Challenges and evidencebased interventions. https://doi.org/10.20529/ IJME.2020.73.

Toral-Villanueva, R., Aguilar-Madrid, G., \& Juárez-Pérez, C. A. (2009). Burnout and patient care in junior doctors in Mexico City. Occupational medicine, 59(1), 8-13. https://doi. org/10.1093/occmed/kqn122 
Artículo empírico | Burnout en profesionales de la salud... Zamudio-Sosa, Rojo-López, Fuentes-Gallegos y Fletes-Gutiérrez

\section{Meta-Análisis del Artículo}




\section{Dimensión Cuantitativa}

\section{Perfil de Evaluación entre pares}
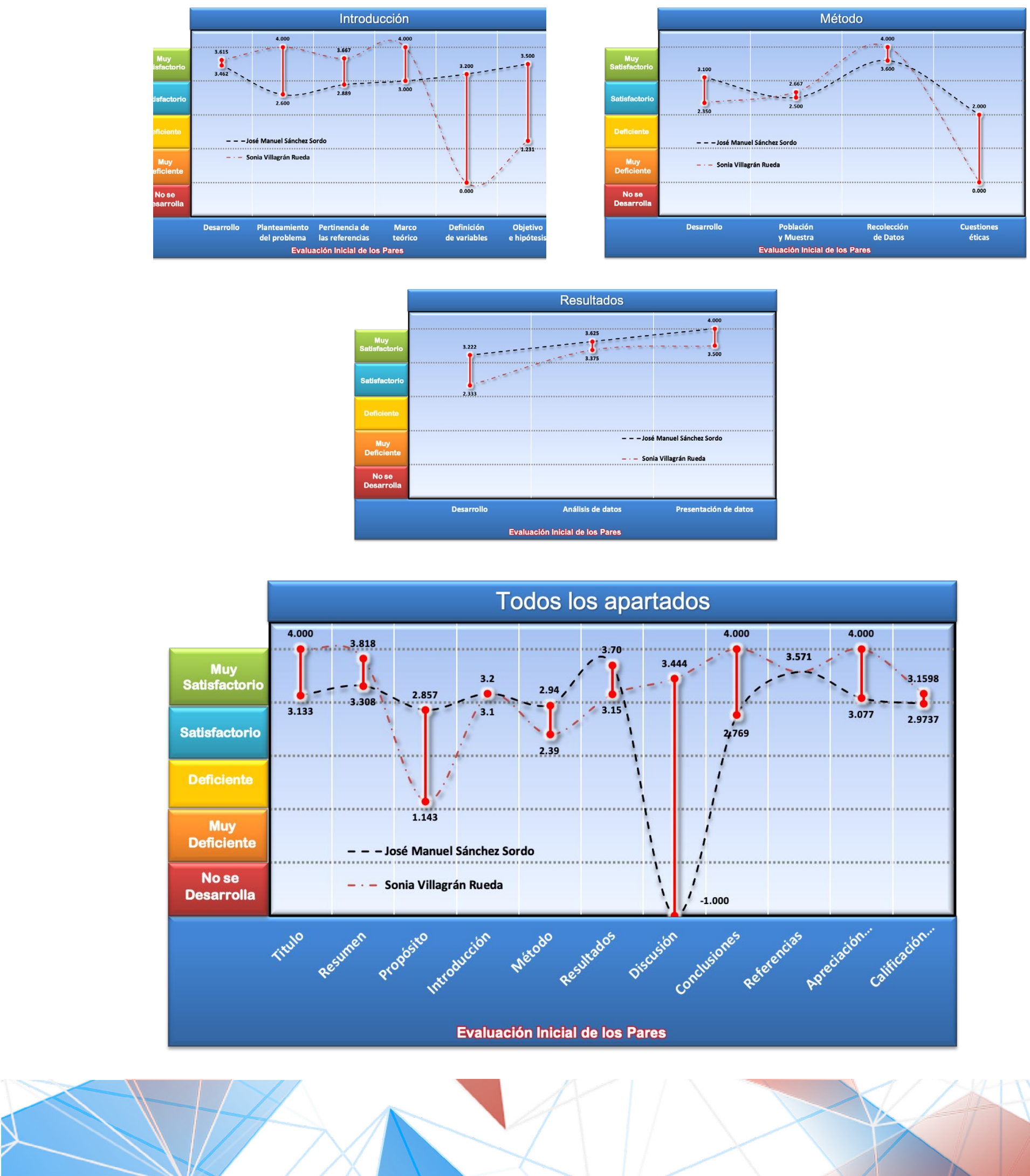


\section{Índice de Concordancia}

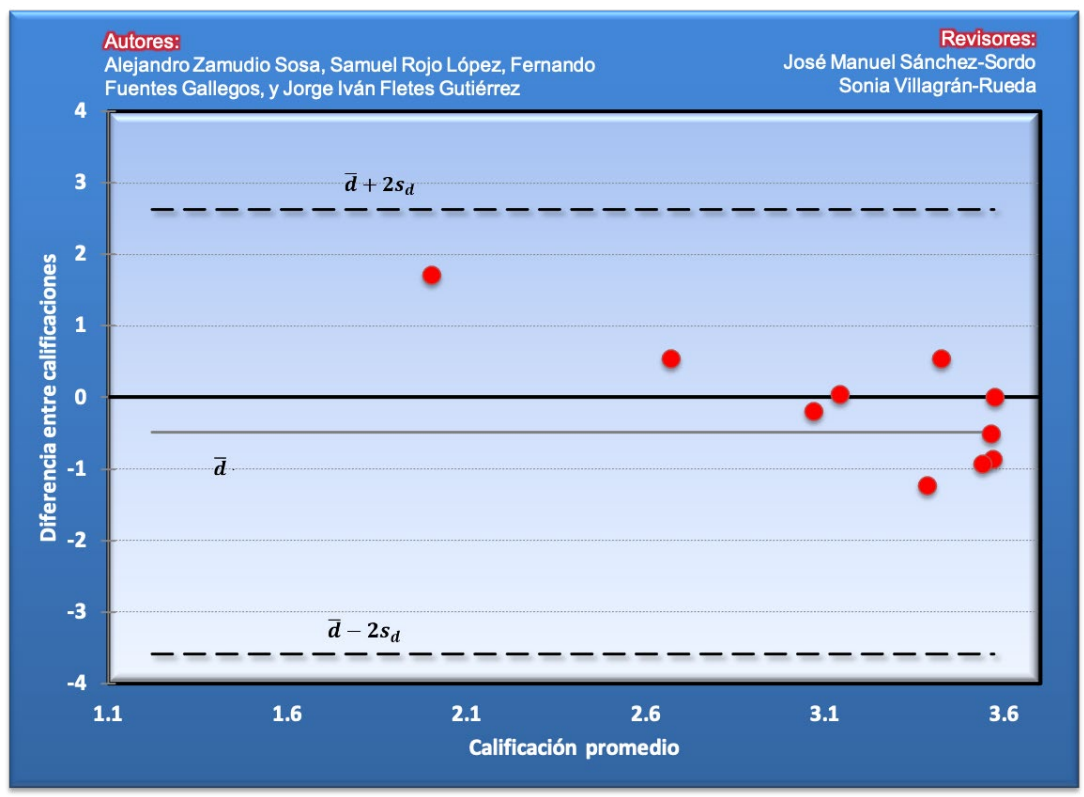

Índice de Acuerdo

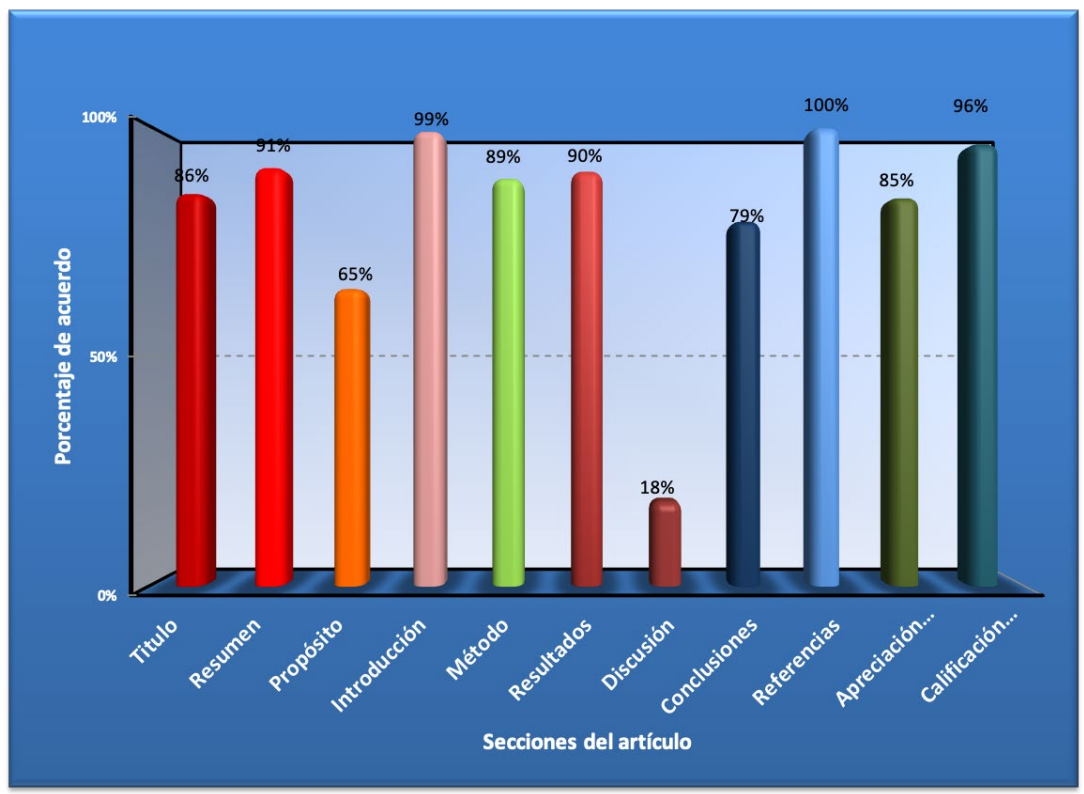




\begin{tabular}{|c|c|}
\hline Revisor 1 & Revisor 2 \\
\hline José Manuel Sánchez Sordo & Sonia Villagrán-Rueda \\
\hline \multicolumn{2}{|c|}{ Título/Autoría } \\
\hline cabría especificar pandemia del Covid-19 & $\begin{array}{l}\text { El título debe ser máximo } 15 \text { palabras, ademas en el abs- } \\
\text { tract falta el título en inglés }\end{array}$ \\
\hline \multicolumn{2}{|c|}{ Resumen } \\
\hline $\begin{array}{l}\text { Incluir un breve resumen de los resultados de los clus- } \\
\text { ters. }\end{array}$ & $\begin{array}{l}\text { El parrafo cuarto se enfocó más a la explicación de pro- } \\
\text { cedimiento y resultados, dejando el parrafo quinto a una } \\
\text { leve descripción de conclusiones, se sugiere ahondar más } \\
\text { en dichas conclusiones. En cuanto al resumen presenta } \\
\text { un total de } 233 \text { palabras, siendo el máximo requerido } 150 \\
\text { palabras, favor de corregir. Las palabras clave o conceptos } \\
\text { son cuatro y los solicitados mínimamente son } 5 \text { palabras } \\
\text { o conceptos clave. Respecto al abstract falta el título en in- } \\
\text { glés, así mismo favor de omitir la indicación de la plantilla } \\
\text { entre corchetes. }\end{array}$ \\
\hline \multicolumn{2}{|c|}{ Próposito del Estudio } \\
\hline $\begin{array}{l}\text { Si bien el propósito es claro, el artículo va indicado o } \\
\text { está contextualizado en la época actual de "pandemia" } \\
\text { y el propósito no retoma dicho contexto, es como si se } \\
\text { hubiera llevado a cabo la investigación fuera del con- } \\
\text { texto del covid 19, mas el título incluye la palabra pan- } \\
\text { demia. }\end{array}$ & $\begin{array}{l}\text { No expresa textualmente preguntas de investigación, ni } \\
\text { hipótesis. Se sugiere plantear preguntas de investigación } \\
\text { o presentar hipótesis de investigación. }\end{array}$ \\
\hline \multicolumn{2}{|c|}{ Introducción } \\
\hline $\begin{array}{l}\text { No se define conceptualmente de manera concreta el bur- } \\
\text { nout, solo se menciona de manera muy breve a manera de } \\
\text { traducción como desgaste, y es la variable de interés por lo } \\
\text { cual se debería definir conceptualmente y sustentar teó- } \\
\text { ricamente. Una vez más no se menciona relación alguna } \\
\text { entre burnout y pandemia, por ende el término pandemia } \\
\text { en el título parece estár de más. }\end{array}$ & $\begin{array}{l}\text { No enuncia como tal una hipótesis, en este caso la lectora } \\
\text { la infiere. }\end{array}$ \\
\hline
\end{tabular}




\begin{tabular}{|l|}
\hline \multicolumn{1}{|c|}{ Revisor 1 } \\
\hline \multicolumn{1}{|c|}{ Mét } \\
\hline El artículo no sigue la estructura de publicación cientí- \\
fica (introducción, método, etc...) si no su propia lógi- \\
ca basada en describir la intervención. Si el objetivo del \\
estudio es clasificar personal de la salud, al haber sido \\
recabada por facebook no hay certeza de que se cuente \\
con una muestra homogénea o con las características de \\
interés, se debió haber buscado directo en hospitales, la- \\
boratorios etc. El instrumento utilizado no se describe, \\
es mas ni se pone su nombre completo, y NO retoman \\
la variable contextual "pandemia" para recabar, analizar \\
y concluir sobre los datos algo relacionado con el estrés \\
laboral y la pandemia.
\end{tabular}

Revisor 2

\section{Resultados}

A nivel de clusterización los resultados son buenos, más éstos no ponen de manifiesto ninguna posible intervención como se menciona en el objetivo del artículo, el clasificador final al parecer tiene buen nivel de clasificación, mas éste es genérico y no está centrado para clasificar estrés en pandemia como el título lo indica...en general hay una desconexión entre e título (pandemia) y los resultados, pues si bien si se encontraron grupos según las variables observadas ninguna de éstas hace referencia directa a la pandemia.

No existe una división de secciones mínimas que señalen Participantes, Materiales, Procedimientos, Mediciones y Análisis estadístico, se sugiere poner dichos apartados para una mejor comprensión. No señala el tipo de muestreo utilizado. No describe si existió algún procedimiento para obtener consentimiento informado, tampoco señala la forma en que aseguró el anonimato y la confidencialidad a los participantes del estudio

Debido a que no plantea tácitamente pregunta de la investigación y/o hipótesis, no se vislumbra en la sección de resultados en aquellos hallazgos pertinentes que respondan a la pregunta de la investigación y/o a la prueba de hipótesis

\section{Discusión}

No hay

No se señalan hipótesis alternativas

\section{Conclusiones}

Se centra mucho en el algoritmo y no en los resultados encontrados con éste.

Las conclusiones son adecuadas.

Referencias

meter más de burnout

Por ejemplo la referencia de Tesillo, S. Y. F., y Martínez, A. O. R. (2018). Burnout en profesionales de la salud y personal administrativo en una unidad médica de primer nivel. Psicología y Salud, 28(1), 63-72, no se encuentra en el documento y si en el listado de referencias. 
